parents are homozygous or hybrid, or are themselves sibs or unrelated? In other words can we disregard the breeding system in estimating the relative effects of genetic and environmental differences on individuals within a family?

(ii) Are all the differences between one-egg twins to be ascribed to differences in the environment? For example, differences due to mirror imaging, to asymmetrical defects such as ptosis, to embryological errors of one or both twins, or to changes of chromosome number or structure such as arise from errors of mitosis at the first or later cleavage divisions.

It is true that these questions did not arise in the mind of Galton, or for the most part in the mind of Fisher, when they first studied correlations between twins and between relatives. It is also true that the attempt to answer them would involve biological study. And the results might then make the data more difficult to handle. But ought we (if I may ask a third question) to ignore what we have come to know in the last forty years in order to arrive at a quick and comfortable answer?

To this very general question it seems that one of the authors, Professor Wiseman, would be prepared to say no. For he suggests that the home is created by parents. The home is therefore not a purely environmental agent: it is connected also with the heredity of children. One can only be surprised that the editors did not delete such an unfashionable suggestion, such an intellectual deviation. For otherwise they have given us 242 pages of discrete conformity.

C. D. Darlington.

\title{
HUMAN ADAPTABILITY
}

THE BIOLOGY OF HUMAN ADAPTABILITY. Edited by P. T. Baker and J. S. Weiner. Clarendon Press, Oxford, 1966. Pp. 541. f5, 5s. Od.

The International Biological Programme, "concerned essentially with the functional relationship of living things to their environments", has, as one of its major projects, world-wide enquiries into the problem of human adaptability. These studies look first, into the extent to which the human genetic system has been moulded to produce heritable adaptation and secondly, into that to which the human phenotype has remained plastic and capable of rapid, non-heritable response to changes of environment. Such work integrated with older anthropometric studies and with analyses of regional variations in the frequency of readily identifiable genetic factors, forms the modern basis for enquiry into variation in extant man.

The impetus imparted to this field by the International Biological Programme, has, as one of its measures, a symposium, held in 1964 and sponsored by the Wenner-Green Foundation, to which a group of 18 workers contributed. Their reviews, transcribed in the present volume, summarise the present state of knowledge of various aspects of human adaptability, both on an international and regional basis.

An initial paper introduces the major problems associated with the comparative physiological and genetic study of human populations, including that of the factors contributing to the production of selective forces. Genetical 
analysis must always play a significant part in such a project and a further paper analyses the design and interpretation of genetic surveys.

Many genetic factors become manifest through their influence on growth processes as also do such selective forces as malnutrition and disease. It is to studies of these that the third contribution is directed.

The capacity for physical work is significant as a partial measure of the biological efficiency of a population, and the fourth contribution to the symposium reviews the relatively restricted amount of information at present bearing on this factor.

The next twelve papers deal with studies of physiological adaptation and of genetic variability in populations from Africa, America, Asia, Australia and the circumpolar regions, while the two final papers bear on fascinating projects relating to adaptability in high altitude populations.

The result is a volume of more than five hundred pages which contains, not only scholarly surveys, but also a mass of detailed factual data of much significance.

Even so, cursory comparison between the contributions relating to populations from different regions of the world indicates forcefully the extent of the information that remains to be collected. But such realisation, in no sense diminishes admiration for the body of work already completed. In the words of Sir Lindor Brown in his foreword to the volume, the International Biological Programme has passed its first inertial stages and "a few rocks and sandbanks of solid achievement are beginning to be visible". In throwing these into sharp relief, the present volume cannot fail to stimulate enthusiasm for their further accretion and eventual amalgamation.

E. H. Ashton.

\section{A PRIMER OF GENETIC METHODOLOGY}

GENETIC ANALYSIS. W. K. Baker. Riverside studies in Biology. Thomas Nelson, London, 1965. Pp. $x+164$. 15s.

In the preface the author makes the point that it has become increasingly unsatisfactory during the last 20 years or so to divide biology into artificial and exclusive compartments called zoology, botany, genetics, etc. Modern biological research is now concentrating on fundamental problems which require a wide spectrum of techniques and experimental organisms for their elucidation. Genetical techniques for example are central in the investigation of such problems as growth and differentiation although these phenomena encompass a good deal of the whole field of biology. Accordingly, the author has written an explanatory account of some of the more important genetical techniques and the kind of answers they provide to certain biological questions.

The general idea of illustrating the genetical approach and giving details of techniques applied to specific problems is a good one. Also the actual choice of topics to be covered within the compass of a small book is good. Chapters of varying length are devoted to: Introduction to genetic analysis; Genetic symbols and ratios in inheritance; Genetic recombinants; Gene action; Mutation; Cytology and analysis of normal and aberrant chromosomes; the Genetic code and finally Cellular Differentiation. There is also 